\title{
ARTICLE
}

\section{Timing of inorganic phosphate release modulates the catalytic activity of ATP-driven rotary motor protein}

Rikiya Watanabe ${ }^{1,2} \&$ Hiroyuki Noji ${ }^{1}$

$\mathrm{F}_{1}$-ATPase is a rotary motor protein driven by ATP hydrolysis. The rotary motion of $\mathrm{F}_{1}$-ATPase is tightly coupled to catalysis, in which the catalytic sites strictly obey the reaction sequences at the resolution of elementary reaction steps. This fine coordination of the reaction scheme is thought to be important to achieve extremely high chemomechanical coupling efficiency and reversibility, which is the prominent feature of $\mathrm{F}_{1}$-ATPase among molecular motor proteins. In this study, we intentionally change the reaction scheme by using single-molecule manipulation, and we examine the resulting effect on the rotary motion of $F_{1}$-ATPase. When the sequence of the products released, that is, ADP and inorganic phosphate, is switched, we find that $F_{1}$ frequently stops rotating for a long time, which corresponds to inactivation of catalysis. This inactive state presents MgADP inhibition, and thus, we find that an improper reaction sequence of $F_{1}$-ATPase catalysis induces MgADP inhibition.

\footnotetext{
${ }^{1}$ Department of Applied Chemistry, School of Engineering, The University of Tokyo, Bunkyo-ku, Tokyo 113-8656, Japan. ${ }^{2}$ PRESTO, JST, Bunkyo-ku, Tokyo 113-8656, Japan. Correspondence and requests for materials should be addressed to H.N. (email: hnoji@appchem.t.u-tokyo.ac.jp).
} 
${ }_{1}$-ATPase $\left(\alpha_{3} \beta_{3} \gamma \delta \varepsilon\right)$, a catalytic sub-complex of $\mathrm{F}_{0} \mathrm{~F}_{1}$-ATP synthase, is a rotary motor protein fuelled by ATP hydrolysis ${ }^{1-3}$. Three subunits $\left(\alpha_{3} \beta_{3} \gamma\right)$ function as the minimum component of the rotating system, where the $\alpha_{3} \beta_{3}$ unit forms a cylindrical stator and the $\gamma$ rotor subunit penetrates the centre of the cylinder ${ }^{4}$. The catalytic sites for ATP hydrolysis are located on each interface between the $\alpha$ and $\beta$ subunits, mainly on the $\beta$ subunits, and the three $\beta$ subunits are always in different catalytic states ${ }^{4}$. The interconversion of the catalytic states of $\beta$ subunits, which induces their conformational transition, drives the rotation of the $\gamma$ subunit $^{5-7}$.

The rotary motion of $\mathrm{F}_{1}$ can be directly visualized by optical microscopy ${ }^{8-10}$. The unitary step size of the rotation of $F_{1}$ is $120^{\circ}$, and each step is coupled to a single turnover of ATP hydrolysis ${ }^{11}$. The $120^{\circ}$ step is further divided into $80^{\circ}$ and $40^{\circ}$ sub-steps ${ }^{12,13}$. The $80^{\circ}$ sub-step is triggered by ATP binding and ADP release, each of which occurs on different $\beta$ subunits ${ }^{14}$. The $40^{\circ}$ sub-step is triggered by ATP hydrolysis and release of inorganic phosphate $\left(\mathrm{P}_{\mathrm{i}}\right)$, which also occurs on different $\beta$ subunits ${ }^{14,15}$. The catalytic state of the $\beta$ subunits is tightly coupled to the rotation of the $\gamma$ subunit; thus, $\mathrm{F}_{1}$ can synthesize ATP from ADP and $\mathrm{P}_{\mathrm{i}}$ when the $\gamma$ subunit is forcibly rotated in the reverse direction ${ }^{16,17}$. Mechanically induced ATP synthesis is the physiological role of $\mathrm{F}_{1}$ of $\mathrm{F}_{0} \mathrm{~F}_{1}$-ATP synthase, in which $\mathrm{F}_{0}$, a proton-driven motor protein, compels $\mathrm{F}_{1}$ to rotate in the reverse direction for ATP synthesis. This reversibility of chemomechanical coupling is the prominent feature of $\mathrm{F}_{1}$ among other motor proteins.

To achieve reversibility of chemomechanical coupling, fine coordination of the reaction sequence, which properly induces the conformational transition of $\beta$ subunits, is required. To understand the fine coordination mechanism, extensive studies have been dedicated to unveiling the basic reaction scheme for the rotation and catalysis of $F_{1}$, focusing on the catalytic turnover of individual $\beta$ subunits (Fig. 1a). In this scheme, an individual $\beta$ subunit binds to ATP when the $\gamma$ subunit is oriented at a specific angle, and the binding angles for the individual $\beta$ subunits differ by $\pm 120^{\circ}$. Each $\beta$ subunit hydrolyses the bound ATP into ADP and $\mathrm{P}_{\mathrm{i}}$ after $\gamma$ rotates another $200^{\circ}$ from the ATP-binding angle $\mathrm{e}^{18}$. In addition, the produced $\mathrm{ADP}$ and $\mathrm{P}_{\mathrm{i}}$ are released from the catalytic site after additional $40^{\circ}$ and $120^{\circ}$ rotations, respectively ${ }^{14,15,19,20}$. When the $\gamma$ subunit returns to the original angular position, the $\beta$ subunit initiates the next round of catalysis by binding to a new ATP. Thus, the reaction sequences among the three $\beta$ subunits are well coordinated at the resolution of elementary reaction steps, which contributes to the reversibility of the chemomechanical coupling on $\mathrm{F}_{1}$.

Then, the question arises as to what happens when the reaction sequence of $\mathrm{F}_{1}$ is changed. In the case of the hydrolysis step, although the timing of hydrolysis shifts by $80^{\circ}$ relative to the authentic reaction angle, $\mathrm{F}_{1}$ drives the rotation without interruption, demonstrating the robustness of the chemomechanical coupling of $\mathrm{F}_{1}$ (ref. 21). In contrast, in the case of other elementary reaction steps, such as $\mathrm{P}_{\mathrm{i}}$ release, the effect of timing on rotary motion has not been examined so far, although we established a method to change the timing of $\mathrm{P}_{\mathrm{i}}$ release by using single-molecule manipulation ${ }^{15}$. In particular, the free energy change resulting from $\mathrm{P}_{\mathrm{i}}$ release was relatively high among reaction steps; that is, the rotary torque was mainly generated by $\mathrm{P}_{\mathrm{i}}$ release, and therefore, the effect of the timing of $\mathrm{P}_{\mathrm{i}}$ release on rotary motion is expected to be larger than that of other reaction steps ${ }^{14,15,22}$. In addition, the timing of $P_{i}$ release in $F_{1}$ is different from that in other motor proteins fuelled by ATP hydrolysis, such as kinesin and myosin, in which $\mathrm{P}_{\mathrm{i}}$ release occurs before ADP release $^{23,24}$, and this difference is thought to be important for the reversibility of the chemomechanical coupling mechanism of $\mathrm{F}_{1}$ (ref. 15).

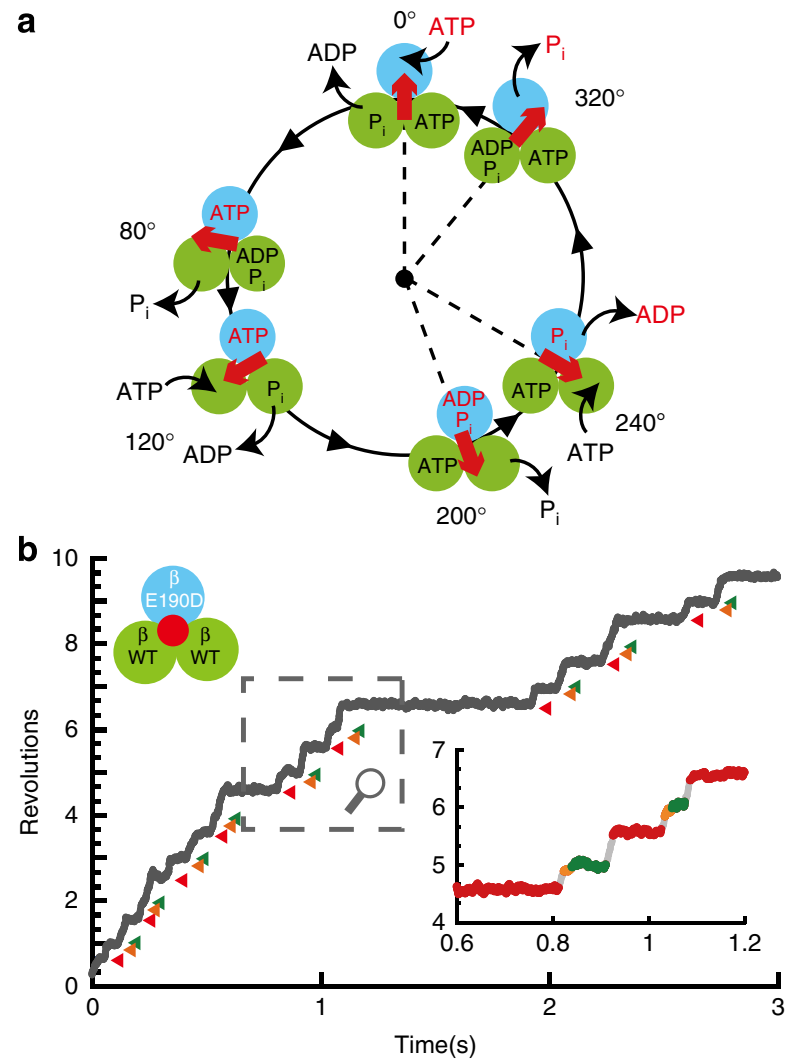

Figure 1 | Chemomechanical coupling scheme of $\mathbf{F}_{\mathbf{1}}$-ATPase. (a) The circles and red arrows represent the catalytic state of the $\beta$ subunits and the angular positions of the $\gamma$ subunit. Each $\beta$ subunit completes one turnover of ATP hydrolysis in a turn of the $\gamma$ subunit, where the three $\beta$ subunits vary in their catalytic phase by $120^{\circ}$. Regarding the catalytic state of the top $\beta$ subunit (cyan), ATP binding, hydrolysis, ADP release and inorganic phosphate $\left(P_{i}\right)$ release occur at $0^{\circ}, 200^{\circ}, 240^{\circ}$ and $320^{\circ}$, respectively. (b) Time course of the rotation of the hybrid $F_{1}$-ATPase, $\alpha_{3} \beta$ (E190D) $\beta_{2} \gamma$, in the presence of $1 \mathrm{mM}$ ATP. Red, orange, and green represent the pause at $200^{\circ}, 320^{\circ}$, and $360^{\circ}$ for $\beta(E 190 D)$, respectively. The inset shows a magnification of the time course.

In this study, to further investigate the role of the finely coordinated reaction sequence of $\mathrm{F}_{1}$, we evaluate the effect of changing the timing of $\mathrm{P}_{i}$ release on the chemomechanical coupling mechanism. When $\mathrm{P}_{\mathrm{i}}$ is released before $\mathrm{ADP}$, we find that $F_{1}$ frequently stops rotating for a long time, which corresponds to inactivation of catalysis. This inactive state presents MgADP inhibition, and thus, we find that an improper timing of $\mathrm{P}_{\mathrm{i}}$ release induces $\mathrm{MgADP}$ inhibition.

\section{Results}

Rotation of hybrid $F_{1}$. We observed the rotation of hybrid $F_{1}$ carrying one $\beta(\mathrm{E} 190 \mathrm{D})$, that is, $\alpha_{3} \beta_{2} \beta(\mathrm{E} 190 \mathrm{D}) \gamma$, in the presence of $1 \mathrm{mM}$ ATP. Glu190 of the $\beta$ subunit, the so-called 'general base', is known to be one of the most important residues for hydrolysis ${ }^{25-27}$, and its substitution into aspartic acid causes distinctively slow hydrolysis of $\mathrm{ATP}^{28}$ and strong temperature sensitivity ${ }^{29}$. Therefore, the kinetic steps of the mutated catalytic site are easily distinguished from those of the other two sites. In the presence of $1 \mathrm{mM}$ ATP, the hybrid $\mathrm{F}_{1}$ showed three distinctive dwells caused by the incorporated $\beta(E 190 D)$; the dwell for the temperature-sensitive reaction at $0^{\circ}(\tau=32 \mathrm{~ms})^{15,29}$, which is the same as the dwell at the ATP-binding angle, ATP hydrolysis at $200^{\circ}(\tau=318 \mathrm{~ms})$, and $\mathrm{P}_{\mathrm{i}}$ release at $320^{\circ}(\tau=9 \mathrm{~ms})$ (cyan in Fig. 1a,b). The dwell time for $\mathrm{P}_{\mathrm{i}}$ release at $320^{\circ}$ was prolonged 

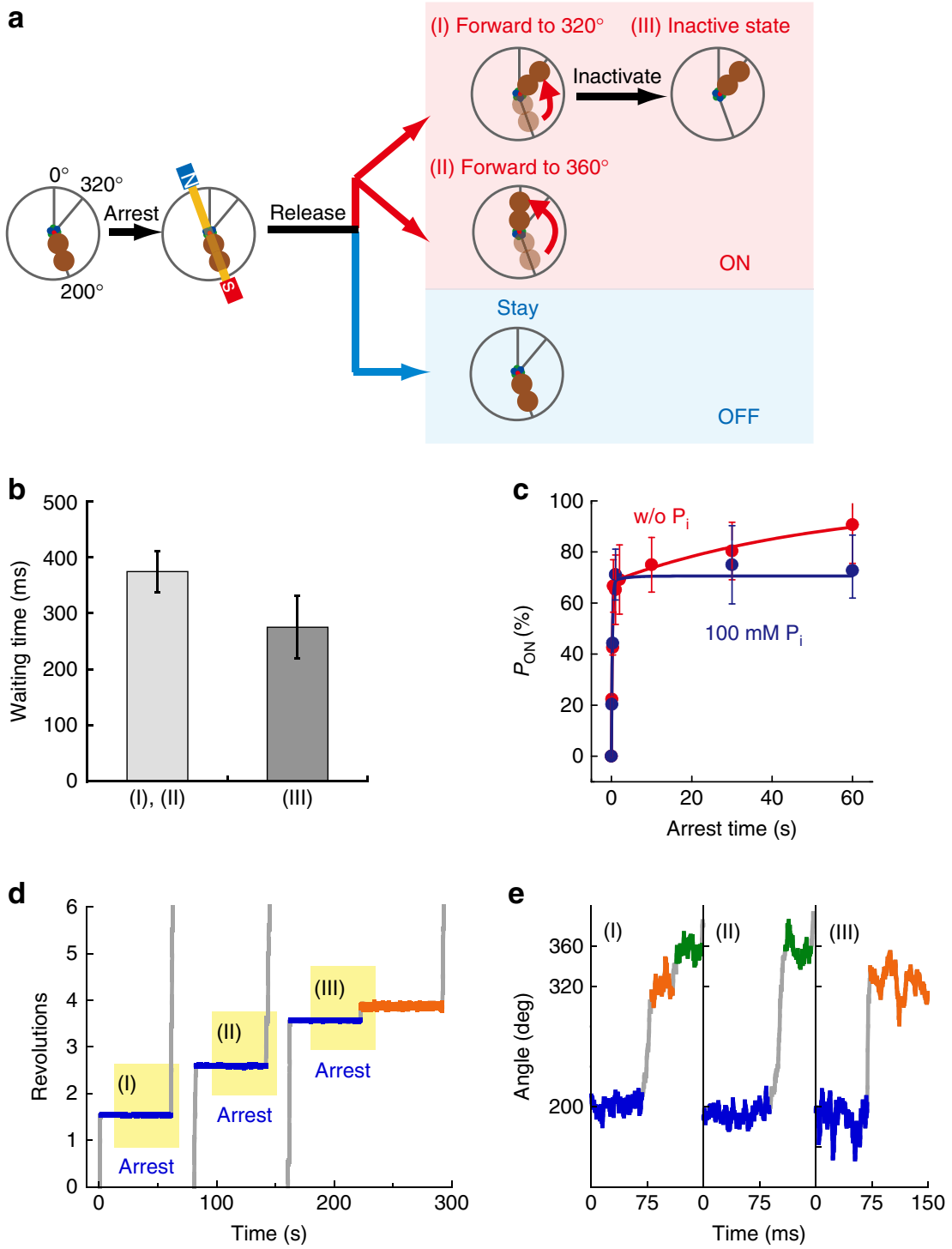

Figure 2 | Single-molecule manipulation for $\mathbf{P}_{\mathbf{i}}$ release. (a) Schematic representation of manipulation procedures. When the hybrid $F_{1}-A T P a s e$ paused at $200^{\circ}$; that is, the angle for ATP hydrolysis by $\beta(E 190 D)$, we switched on the magnetic tweezers and arrested $F_{1}$ at $\pm 10^{\circ}$ from $200^{\circ}$. After release from arrest, $\mathrm{F}_{1}$-ATPase roughly showed two behaviours: rotating forward to next reaction angle ('ON'), or staying at the original pausing angle ('OFF'). In addition, the behaviour of ' $O N^{\prime}$ ' was classified into three types: (I) rotating forward to $320^{\circ}$ and showing the distinctive pause for $\mathrm{P}_{\mathrm{i}}$ release (time constant ( $\tau$ ) is $\sim 9 \mathrm{~ms}$ ); (II) rotating forward to $360^{\circ}$ (skip the pause at $320^{\circ}$ ); or (III) rotating forward to $320^{\circ}$ and showing a long pause $(\tau=\sim 39 \mathrm{~s}$ ).

(b) Hydrolysis-waiting time at $200^{\circ}$ before $\mathrm{F}_{1}$-ATPase is arrested with the magnetic tweezers. The average waiting time was $370 \pm 37 \mathrm{~ms}$ for the behaviours (I) and (II) (light grey), and $275 \pm 56 \mathrm{~ms}$ for the behaviour (III) (grey). Error bars indicate s.d. (c) Time course of $P_{\mathrm{ON}}$. Red and blue points represent $P_{\mathrm{ON}}$ in the absence or presence of $100 \mathrm{mM} \mathrm{P}$, respectively. The number of arrest trials and molecules used for each data point were $23-108$ and 8-19. The time courses were fitted with the reaction scheme; $F_{1} \cdot A T P \leftrightarrow \cdot F_{1}\left(A D P+P_{i}\right) \rightarrow F_{1} \cdot A D P+P_{i}$ (solid lines). (d) Example of the time course of the arrest experiments (blue periods) corresponding to the behaviour of (I), (II) and (III), respectively. (e) Magnification of $\mathbf{d}$. Orange and green represent the pauses at $320^{\circ}$ and $360^{\circ}$.

because of the viscous drag of the rotary probe on $\gamma$ as previously reported $^{30,31}$. Hereafter, we refer to the angle for ATP hydrolysis by $\beta(\mathrm{E} 190 \mathrm{D})$ as the catalytic angle.

Single-molecule manipulation to induce $P_{i}$ release. During free rotation, one $\beta$ hydrolyses ATP and another $\beta$ releases the produced $P_{i}$ at the catalytic angle, resulting in a rotation of the $\gamma$ subunit in an anticlockwise direction (Fig. 1a). In contrast to the free rotation, we recently succeeded in inducing the release of another produced $\mathrm{P}_{\mathrm{i}}$ from the same catalytic site used for ATP hydrolysis by arresting the $\gamma$ subunit for a long time $(>10 s)^{15,22}$.
In this study, we used the same method to induce the release of produced $\mathrm{P}_{\mathrm{i}}$ immediately after ATP hydrolysis. For manipulation of $\gamma$ rotation, a magnetic bead was attached to the $\gamma$ subunit of $F_{1}$, and the $\alpha_{3} \beta_{3}$ ring was immobilized on the glass surface. When the hybrid $\mathrm{F}_{1}$ showed a pause for ATP hydrolysis by $\beta(\mathrm{E} 190 \mathrm{D})$ at $200^{\circ}$, we turned on the magnetic tweezers within $\sim 450 \mathrm{~ms}$ (Fig. 2a,b) and arrested $\mathrm{F}_{1}$ at $\pm 10^{\circ}$ from $200^{\circ}$. After a certain time period had elapsed, we turned off the magnetic tweezers and released $F_{1}$ from arrest. Then, $F_{1}$ roughly showed one of two behaviours without exception, as previously reported: rotating forward or restarting the hydrolysis-waiting pause (Fig. 2a). When $F_{1}$ shows the former behaviour, $\beta(E 190 D)$ has already 

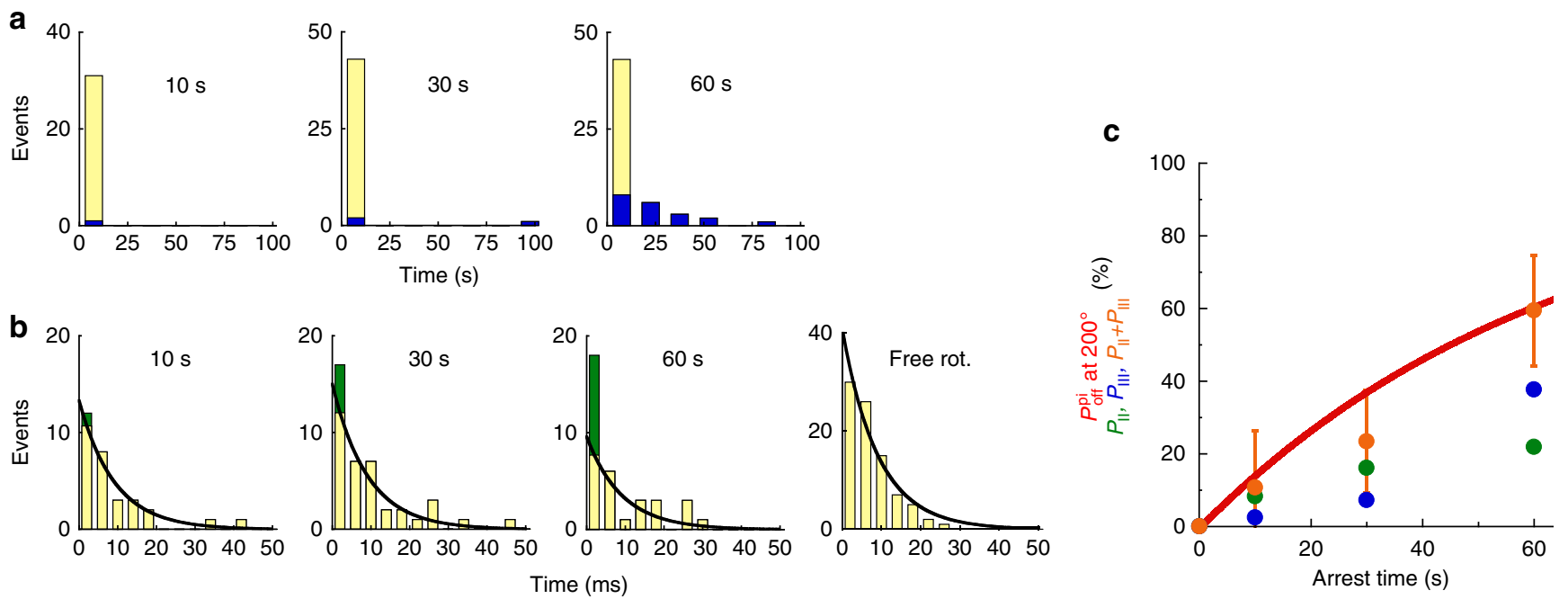

Figure 3 | Analysis of the effect of $\mathbf{P}_{\mathbf{i}}$ release at $\mathbf{2 0 0}^{\circ}$. (a,b) Histograms of the dwell time at $320^{\circ}$ after the release from $200^{\circ}$ arrest for $10 \mathrm{~s}, 30 \mathrm{~s}$, and $60 \mathrm{~s}$, or during free rotation. The bin sizes of the histogram are $15 \mathrm{~s}$ for $\mathbf{a}$ and $4 \mathrm{~ms}$ for $\mathbf{b}$. Blue bars in a represent the number of pauses longer than $100 \mathrm{~ms}$ (behaviour (III)). The data after first bin in $\mathbf{b}$ were well-fitted by the single exponential curve where $\tau=9 \mathrm{~ms}\left(P_{\mathrm{i}}\right.$ release dwell at $\left.320^{\circ}\right)$. The deviations of the first bins from the single exponential curves are coloured green (behaviour (II)). (c) Probability of $P_{i}$ release at $200^{\circ}$. The red solid line presents the simulation curve for $P_{i}$ release calculated from the analysis of $P_{\mathrm{ON}}$ based on the kinetic scheme as follows: $F_{1} \cdot A T P \leftrightarrow F_{1} \cdot\left(A D P+P_{i}\right) \rightarrow$ $\mathrm{F}_{1} \cdot \mathrm{ADP}+\mathrm{P}_{\mathrm{i}}$. Green, blue and orange points represent the probability of occurrence of $\|\left(P_{\|}\right)$, III $\left(P_{\| I I}\right)$ and their sum $\left(P_{\| \mid}+P_{\| I I}\right)$, respectively. $P_{\| \mid}$and $P_{\| I I}$ were determined from the analysis of $\mathbf{b}$ and $\mathbf{a}$, respectively.
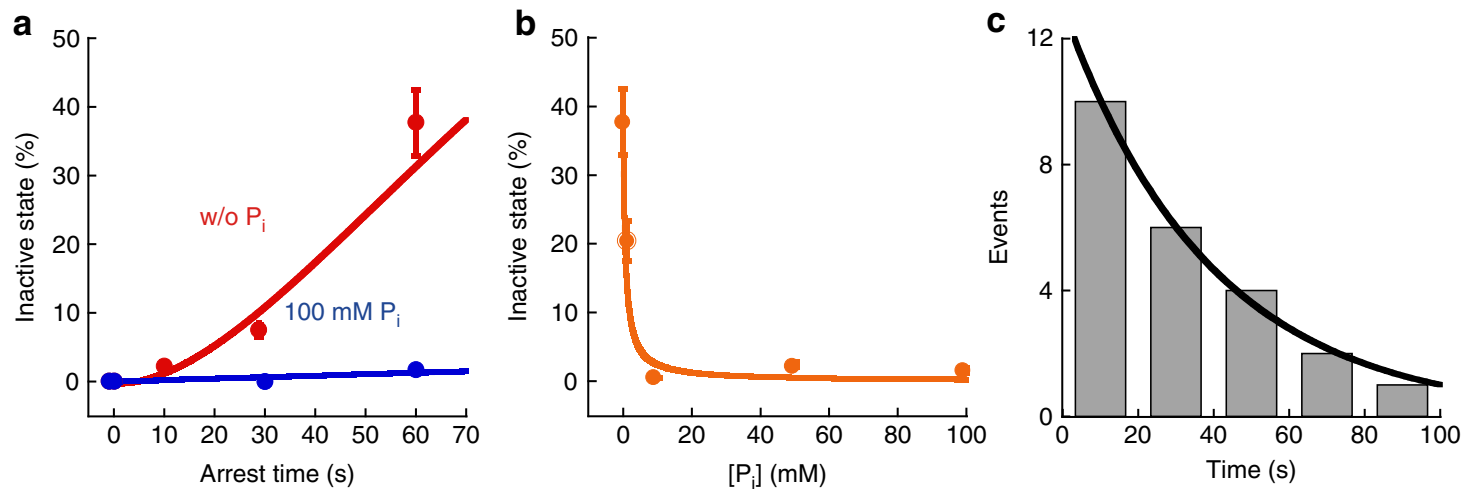

Figure 4 | Analysis of the long pause at $\mathbf{3 2 0}^{\circ}$. (a) Time course of $P_{\mathrm{III}}$ in the absence (red) or presence of $100 \mathrm{mM} \mathrm{P}_{\mathrm{i}}$ (blue). The red solid line is the fitting curve based on the kinetic scheme [1]. The blue solid line is the simulation curve based on the kinetic parameters determined from the fitting of $P_{I I I}$ in the absence of $P_{\mathrm{i}}$. (b) Relationship between $P_{I \mid I}$ for $60 \mathrm{~s}$ arrest and $\mathrm{P}_{\mathrm{i}}$ concentration. The simulation curve in Fig. $4 \mathrm{~b}$ was redrawn against the $P_{i}$ concentration. (c) Histogram of durations of the long pauses at $320^{\circ}$ from the experiments of $10-60 \mathrm{~s}$ arrests. Solid line represents the fitting with single exponential curve where the time constant $\left(\tau_{i \rightarrow a}\right)$ is $39 \mathrm{~s}$. Bin size is $20 \mathrm{~s}$.

hydrolysed ATP and exerted a torque on the magnetic beads, whereas when $\beta(\mathrm{E} 190 \mathrm{D})$ shows the latter behaviour, it has not hydrolysed ATP because $\mathrm{F}_{1}$ cannot generate torque unless $\beta(\mathrm{E} 190 \mathrm{D})$ catalyses hydrolysis. These behaviours are hereafter referred to as 'on' and 'off, respectively. Then, we measured the probability of the post-hydrolysis state as the probability of the 'on' case against the total number of trials, $P_{\mathrm{ON}}$. In the absence of $P_{\mathrm{i}}$ in solution, $P_{\mathrm{ON}}$ increased toward $100 \%$ as the arrest time increased; in contrast, in the presence of $100 \mathrm{mM} \mathrm{P}_{\mathrm{i}}, P_{\mathrm{ON}}$ did not change depending on the arrest time, which suggests that $P_{\mathrm{ON}}$ depends on the time course of $P_{i}$ release from the catalytic site (Fig. 2c).

We extensively analysed the 'on' case and found that it can be classified into three behaviours; (I) rotating forward to $320^{\circ}$, (II) rotating forward to $360^{\circ}$; that is, skipping the pause at $320^{\circ}$, and (III) rotating forward to $320^{\circ}$ and spontaneously stopping the rotation for a long time (Fig. 2d,e). When $\beta($ E190D) shows (I), it has hydrolysed ATP during its arrest but has not released the produced $\mathrm{P}_{\mathrm{i}}$ because the rate-limiting step at $320^{\circ}$ is not hydrolysis by $\beta(\mathrm{WT})$ but rather $\mathrm{P}_{\mathrm{i}}$ release by $\beta(\mathrm{E} 190 \mathrm{D})$ (Fig. 1a). $\mathrm{F}_{1}$ cannot generate torque at $320^{\circ}$ unless $\beta(\mathrm{E} 190 \mathrm{D})$ releases $\mathrm{P}_{\mathrm{i}}$. When $\beta($ E190D) shows (II), it has hydrolysed ATP and simultaneously released the produced $\mathrm{P}_{\mathrm{i}}$, and therefore, $\mathrm{F}_{1}$ has already exerted torque for the step from $320^{\circ}$ to $360^{\circ}$ driven by $\mathrm{P}_{\mathrm{i}}$ release. Regarding the behaviour of (III), we have not identified the chemical state of $\beta(E 190 D)$ thus far; however, the pausing angle is the same as that of the MgADP-inhibited state ${ }^{32}$. Hereafter, we characterize (III). First, we analysed the dwell time at $320^{\circ}$ and calculated the probability of occurrence for each behaviour. As shown by the blue bars in Fig. 3a, distinctively long dwell times (longer than $100 \mathrm{~ms}$ ), which correspond to the behaviour of (III), can be recognized. Then, we calculated the probability of occurrence of (III), that is, $P_{\mathrm{III}}$, as the number of events corresponding to the blue bars divided by total number of 


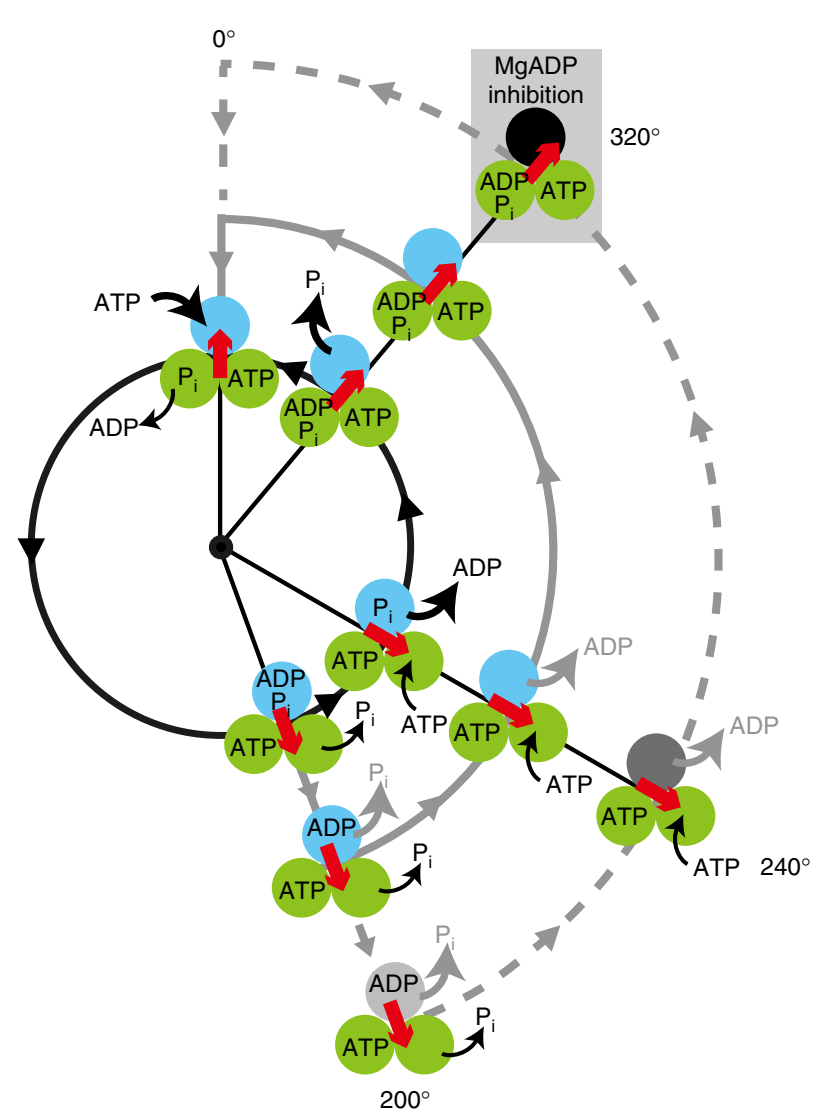

Figure 5 | Mechanism of MgADP-inhibited state. Black solid line: the normal reaction pathway of active $F_{1}$-ATPase. $F_{1}$-ATPase releases $P_{i}$ at $320^{\circ}$. Grey lines: alternative reaction pathways. $F_{1}$-ATPase releases $P_{i}$ before ADP at $200^{\circ}$. $\mathrm{F}_{1}$-ATPase lapses into MgADP inhibition at $320^{\circ}$ with some probability (dashed line).

events in the histograms, and found that $P_{\mathrm{III}}$ increased as the arrest time increased. As shown in Fig. 3b, we changed the time scale of the histogram to shorter bins ( $4 \mathrm{~ms}$ ), and found that the histogram deviated from a single exponential decay model as the arrest time increased. For example, at $60 \mathrm{~s}$ of arrest, the first bin $(<4 \mathrm{~ms})$ contained many more events in which $\beta(\mathrm{E} 190 \mathrm{D})$ skipped the pause at $320^{\circ}$ than expected from a single exponential decay that fit the data well after the first bin with a time constant of $9 \mathrm{~ms}$ (black line in Fig. 3b), which was consistent with that of $\mathrm{P}_{\mathrm{i}}$ release in free rotation (rightmost panel in Fig. 3b). Therefore, the first bin deviated from single exponential decay because of the behaviour of (II); accordingly, the first bin potentially includes behaviours of both (I) and (II). To calculate the probability of occurrence of (II), that is, $P_{\mathrm{II}}$, we fit the data points of the histograms (except for the first bin) to a single exponential curve with $9 \mathrm{~ms}$ as the time constant of $\mathrm{P}_{\mathrm{i}}$ release. $P_{\mathrm{II}}$ was determined as the number of events that deviated from the single exponential curve in the first bin (green bars in Fig. 3b) divided by total number of events of the histogram in Fig. 3a. In addition, we also calculated the probability of occurrence of (I), that is, $P_{\mathrm{I}}$, as the number of remaining events (light yellow bars in Fig. $3 \mathrm{~b}$ ) divided by total number of events.

Kinetic analysis of $\mathbf{P}_{\mathbf{i}}$ release and the inactive state. From the analysis of $P_{\mathrm{ON}}$ based on the irreversible reaction scheme (Fig. 2c) $\mathrm{F}_{1} \cdot \mathrm{ATP} \leftrightarrow \mathrm{F}_{1} \cdot\left(\mathrm{ADP}+\mathrm{P}_{\mathrm{i}}\right) \rightarrow \mathrm{F}_{1} \cdot \mathrm{ADP}+\mathrm{P}_{\mathrm{i}}$, we can calculate the probability of $\mathrm{P}_{\mathrm{i}}$ release during arrest as $P_{\mathrm{off}}^{\mathrm{Pi}}$, as shown by the red line in Fig. 3c. For example, $P_{\text {off }}^{\mathrm{Pi}}$ is $37 \%$ and $60 \%$ for $30-s$ and 60-s arrest, respectively. Here, we compared $P_{\mathrm{II}}$ and $P_{\mathrm{off}}^{\mathrm{Pi}}$, and found that $P_{\mathrm{II}}$ was much smaller than $P_{\text {off }}^{\mathrm{Pi}}$ (Fig. $3 \mathrm{c}$, green). As mentioned above, we could not identify the chemical state of (III). Thus, we suspected that (III) was caused by $\mathrm{P}_{\mathrm{i}}$ release immediately after ATP hydrolysis and summed $P_{\mathrm{II}}$ and $P_{\mathrm{III}}$. The sum of $P_{\mathrm{II}}$ and $P_{\mathrm{III}}$ was almost the same as $P_{\text {off }}^{\mathrm{Pi}}$ (Fig. $3 \mathrm{c}$, orange), suggesting that (III) was caused by $\mathrm{P}_{\mathrm{i}}$ release immediately after ATP hydrolysis. To confirm the relationship between (III) and $\mathrm{P}_{\mathrm{i}}$ release, we conducted the arrest measurement in the presence of high $\left[\mathrm{P}_{\mathrm{i}}\right](\sim 100 \mathrm{mM})$, where $\mathrm{P}_{\mathrm{i}}$ did not affect the kinetics of ATP binding and produced ADP release ${ }^{14,33}$. When we added more than $10 \mathrm{mM} \mathrm{P} \mathrm{P}_{\mathrm{i}}$ in solution, $P_{\mathrm{III}}$ was completely suppressed toward $0 \%$ (Fig. 4a,b), suggesting that (III) was induced after the $\mathrm{P}_{\mathrm{i}}$ release. Then, the kinetic scheme of (III) is given below (equation (1)), where, $k_{\mathrm{hyd}}^{\mathrm{ATP}}, k_{\text {syn }}^{\mathrm{ATP}}, k_{\mathrm{off}}^{\mathrm{Pi}}, k_{\mathrm{on}}^{\mathrm{Pi}}$, and $k_{\mathrm{a} \rightarrow \mathrm{i}}$ are the rate constants of ATP hydrolysis, synthesis, $\mathrm{P}_{\mathrm{i}}$ release, binding and inactivation of $\beta(\mathrm{E} 190 \mathrm{D})$, respectively.

$$
\begin{aligned}
\mathrm{F}_{1} \cdot \mathrm{ATP} & \underset{k_{\text {syn }}^{\text {ATP }}}{\stackrel{k_{\text {hyd }}^{A T P}}{\rightleftarrows}} \mathrm{F}_{1} \cdot\left(\mathrm{ADP}+\mathrm{P}_{\mathrm{i}}\right) \underset{k_{o n}^{P i}}{\stackrel{k_{o f f}^{P i}}{\rightleftarrows}} \mathrm{F}_{1} \cdot \mathrm{ADP}+\mathrm{P}_{\mathrm{i}} \\
& \stackrel{k_{a \rightarrow i}}{\longrightarrow} \mathrm{F}_{1} \cdot \mathrm{ADP}^{*}+\mathrm{P}_{\mathrm{i}}
\end{aligned}
$$

As previously reported, $k_{\text {hyd }}^{\mathrm{ATP}}, k_{\text {syn }}^{\mathrm{ATP}}, k_{\text {off }}^{\mathrm{Pi}}$, and $k_{\text {on }}^{\mathrm{Pi}}$ were given as $2.3 \mathrm{~s}^{-1}, 0.8 \mathrm{~s}^{-1}, 0.021 \mathrm{~s}^{-1}$ and $2 \times 10^{3} \mathrm{M}^{-1} \mathrm{~s}^{-1}$, respectively ${ }^{15}$. Then, from the fitting of $P_{\mathrm{III}}$ in Fig. $4 \mathrm{a}$ based on the kinetic scheme as shown above, the only fitting parameter; $k_{\mathrm{a} \rightarrow \mathrm{i}}$, was determined as $0.019 \mathrm{~s}^{-1}$. To verify this kinetic scheme, we fixed the arrest time as $60 \mathrm{~s}$, and we re-plotted $P_{\text {III }}$ against $\mathrm{P}_{\mathrm{i}}$ concentration. Based on this kinetic scheme with the determined $k_{\mathrm{a} \rightarrow \mathrm{i}}$, we mathematically reproduced the experimental result of $P_{\text {III }}$ as shown in Fig. 4 b. From these results, we confirmed that this inactive state was induced after $P_{i}$ release at $200^{\circ}$ with a rate constant of $0.019 \mathrm{~s}^{-1}$.

We analysed the dwell time for activation from this inactive state (Fig. 4c). As shown in Fig. 4c, the histogram of the dwell time fit well to a single exponential curve, and we determined the time constant of activation, $\tau_{\mathrm{i} \rightarrow \mathrm{a}}$, as $39 \mathrm{~s}$, which was almost the same as that for activation from an MgADP-inhibited state ${ }^{32}$. In addition, the aforementioned phenomena in which the addition of a large amount of $\mathrm{P}_{\mathrm{i}}$ prohibited $\mathrm{F}_{1}$ from lapsing into the inactive state (Fig. 4a) is a well-known feature of the MgADPinhibited state ${ }^{34}$, suggesting that the inactive state revealed in this study corresponds to MgADP inhibition.

\section{Discussion}

MgADP inhibition, a common feature of $F_{1}$-ATPase and ATP synthases from various sources ${ }^{32,34-37}$, is the catalytically inactive state. Although many biochemical studies have addressed MgADP inhibition, the mechanism of MgADP inhibition has remained elusive in the context of catalysis-rotation scheme. Here, we propose a simple model for how $\mathrm{F}_{1}$ lapses into the MgADP-inhibited state during catalysis (Fig. 5). We assume that the fundamental principle of MgADP inhibition is the loss of the driving energy of rotation derived from $\mathrm{P}_{\mathrm{i}}$ release. Our experimental data indicate that $\mathrm{F}_{1}$ can continue rotating even if it releases $P_{i}$ before $A D P$ at $200^{\circ}$, suggesting that $F_{1}$ principally stores the driving energy derived from $\mathrm{P}_{\mathrm{i}}$ release in conformation for $320^{\circ}-360^{\circ}$ rotation (grey solid line in Fig. 5). However, with a time constant of $0.019 \mathrm{~s}^{-1}, \mathrm{~F}_{1}$ dissipates the stored energy during rotation before reaching $320^{\circ}$ and lapses into MgADP inhibition at $320^{\circ}$ (grey dash line in Fig. 5). The driving force of $320-360^{\circ}$ rotation after inhibition is a thermal agitation, as our previous work suggests that thermally agitated rotation of $\gamma$ over $30^{\circ}$ induced activation from the MgADP-inhibited state ${ }^{37}$. Thus, the 
MgADP-inhibited state is so stable that it takes a long time to resume rotation, $\tau_{\mathrm{i} \rightarrow \mathrm{a}}=39 \mathrm{~s}$ (Fig. $\left.4 \mathrm{c}\right)^{32}$.

The crystal structures co-crystallized with azide, including the original structure ${ }^{4,38}$, revealed that $\beta$ at $320^{\circ}\left(\beta_{\mathrm{E}}\right)$ does not bind nucleotides or phosphate. Considering that azide is well known to stabilize the MgADP-inhibited state of $\mathrm{F}_{1}$, these structures demonstrate the structural features of the MgADP-inhibited state. Thus, it is highly probable that $\beta$ in the $320^{\circ}$ state of MgADPinhibited $F_{1}$ has no bound nucleotide or phosphate, which is consistent with our model (Fig. 5).

It should be mentioned that the Walker group has challenged our reaction scheme of catalysis and rotation for active $F_{1}$, on which the present work is based, that is, where $P_{i}$ is released from $\beta$ in $320^{\circ}$ state. Based on the recent crystal structure of $\mathrm{MF}_{1}$ in which $\beta$ in the $320^{\circ}$ state is bound to $\mathrm{Mg}$-free $\mathrm{ADP}^{39}$, they proposed that $\mathrm{Mg}$ and $\mathrm{P}_{\mathrm{i}}$ are released before ADP. Although the reason for this apparent discrepancy is not clear, it should be noted that most of the crystal structures solved so far had no bound nucleotide on $\beta$ in the $320^{\circ}$ state; the only exceptions are the above-mentioned structure $^{39}$ and a structure in a different study ${ }^{40}$. In addition, there are several experimental differences between the present study and the crystal structure study such as the species from which $F_{1}$ is derived and the buffer contents. A precise correlation between the crystal structure and the reaction model based on single-molecule experiments remains to be established.

In vivo, $\mathrm{F}_{1}$ forms $\mathrm{F}_{0} \mathrm{~F}_{1}$ - ATP synthase and synthesizes ATP by coupling with a rotary motion driven by $\mathrm{F}_{0}$. Previous studies revealed that MgADP inhibition suppresses ATP hydrolysis but not synthesis ${ }^{41}$. Recently, the rotary motion of the $\mathrm{F}_{0} \mathrm{~F}_{1}$ complex coupled to the synthesis of ATP was visualized at the single-molecule level ${ }^{42-}$ 44 . For further understanding of the effect of MgADP inhibition on ATP synthesis, verification by single-molecule measurement of the $\mathrm{F}_{0} \mathrm{~F}_{1}$ complex is desirable in the future.

\section{Methods}

Rotation assay. To visualize the rotation of hybrid $\mathrm{F}_{1}, \alpha_{3} \beta$ (E190D) $\beta_{2} \gamma$, the stator region $\left(\alpha_{3} \beta(E 190 D) \beta_{2}\right)$ was fixed on the glass surface, and a magnetic bead $(\phi=0.3 \mu \mathrm{m}$; Seradyn, USA) was attached to the rotor part $(\gamma)$ as a probe for rotation and manipulation. The experimental procedure was as follows. The flow chamber was constructed from an uncoated top coverslip and a bottom coverslip whose surface was modified with $\mathrm{Ni}^{2+} \mathrm{NTA}$. $\mathrm{F}_{1}$ solution, which was diluted in buffer A ( $50 \mathrm{mM}$ MOPS-KOH, $50 \mathrm{mM} \mathrm{KCl}$ and $5 \mathrm{mM} \mathrm{MgCl}_{2}, \mathrm{pH} 7.0$ ) to a final concentration of $200 \mathrm{pM}$, was infused into the flow chamber. After $5 \mathrm{~min}$, unbound $\mathrm{F}_{1}$ molecules were washed out with buffer A containing $10 \%$ bovine serum albumin, and then streptavidin-coated magnetic beads in buffer A were infused. After $10 \mathrm{~min}$, unbound beads were washed out with buffer A. Finally, buffer A was infused with the indicated amount of ATP or $\mathrm{P}_{\mathrm{i}}$. The rotating beads were observed under a phase-contrast microscope (IX-70 or IX-71; Olympus, Japan) with a $\times 100$ objective lens. The rotation assay was performed at $23^{\circ} \mathrm{C}$.

Manipulation with magnetic tweezers. The stage of the microscope was equipped with magnetic tweezers that were controlled using custom-made software (Celery, Library, Japan). The rotary motion of the bead was recorded at 30 and 3,000 frames per second, simultaneously (FC300M, Takex, Japan; FASTCAM 1024PCI-SE, Photron, Japan). Images were stored in the hard disk drive of a computer as an AVI file and analysed using custom-made software. From the recorded images at 3,000 frames per second, we judged whether the operations precisely arrested the $\gamma$ subunit at the targeted angle or not.

\section{References}

1. Yoshida, M., Muneyuki, E. \& Hisabori, T. ATP synthase-a marvellous rotary engine of the cell. Nat. Rev. Mol. Cell Biol. 2, 669-677 (2001).

2. Junge, W., Sielaff, H. \& Engelbrecht, S. Torque generation and elastic power transmission in the rotary $\mathrm{F}_{\mathrm{o}} \mathrm{F}_{1}$-ATPase. Nature 459, 364-370 (2009).

3. Weber, J. Structural biology: Toward the ATP synthase mechanism. Nat. Chem. Biol. 6, 794-795 (2010).

4. Abrahams, J. P., Leslie, A. G., Lutter, R. \& Walker, J. E. Structure at $2.8 \AA$ resolution of $\mathrm{F}_{1}$-ATPase from bovine heart mitochondria. Nature 370, 621-628 (1994).

5. Wang, H. \& Oster, G. Energy transduction in the $F_{1}$ motor of ATP synthase. Nature 396, 279-282 (1998)
6. Boyer, P. D. The binding change mechanism for ATP synthase-some probabilities and possibilities. Biochim. Biophys. Acta 1140, 215-250 (1993).

7. Mukherjee, S. \& Warshel, A. Electrostatic origin of the mechanochemical rotary mechanism and the catalytic dwell of $\mathrm{F}_{1}$-ATPase. Proc. Natl Acad. Sci. USA 108, 20550-20555 (2011).

8. Noji, H., Yasuda, R., Yoshida, M. \& Kinosita, Jr. K. Direct observation of the rotation of $F_{1}$-ATPase. Nature 386, 299-302 (1997).

9. Spetzler, D. et al. Microsecond time scale rotation measurements of single $\mathrm{F}_{1}$-ATPase molecules. Biochemistry 45, 3117-3124 (2006).

10. Panke, O., Cherepanov, D. A., Gumbiowski, K., Engelbrecht, S. \& Junge, W. Viscoelastic dynamics of actin filaments coupled to rotary F-ATPase: angular torque profile of the enzyme. Biophys. J. 81, 1220-1233 (2001).

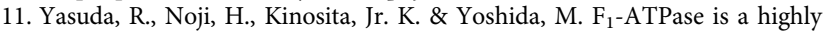
efficient molecular motor that rotates with discrete 120 degree steps. Cell 93, 1117-1124 (1998).

12. Yasuda, R., Noji, H., Yoshida, M., Kinosita, Jr. K. \& Itoh, H. Resolution of distinct rotational substeps by submillisecond kinetic analysis of $F_{1}$-ATPase. Nature 410, 898-904 (2001).

13. Shimabukuro, K. et al. Catalysis and rotation of $\mathrm{F}_{1}$ motor: cleavage of ATP at the catalytic site occurs in $1 \mathrm{~ms}$ before 40 degree substep rotation. Proc. Natl Acad. Sci. USA 100, 14731-14736 (2003).

14. Adachi, K. et al. Coupling of rotation and catalysis in $\mathrm{F}_{1}-\mathrm{ATP}$ ase revealed by single-molecule imaging and manipulation. Cell 130, 309-321 (2007).

15. Watanabe, R., Ino, R. \& Noji, H. Phosphate release in $F_{1}$-ATPase catalytic cycle follows ADP release. Nat. Chem. Biol. 6, 814-820 (2010).

16. Itoh, H. et al. Mechanically driven ATP synthesis by $\mathrm{F}_{1}$-ATPase. Nature 427, 465-468 (2004).

17. Rondelez, Y. et al. Highly coupled ATP synthesis by $F_{1}-A T P a s e$ single molecules. Nature 433, 773-777 (2005).

18. Ariga, T., Muneyuki, E. \& Yoshida, M. F F $^{-A T P a s e}$ rotates by an asymmetric, sequential mechanism using all three catalytic subunits. Nat. Struct. Mol. Biol. 14, 841-846 (2007).

19. Nishizaka, T. et al. Chemomechanical coupling in $\mathrm{F}_{1}$-ATPase revealed by simultaneous observation of nucleotide kinetics and rotation. Nat. Struct. Mol. Biol. 11, 142-148 (2004).

20. Okazaki, K. \& Hummer, G. Phosphate release coupled to rotary motion of $F_{1}$-ATPase. Proc. Natl Acad. Sci. USA 110, 16468-16473 (2013).

21. Shimabukuro, K., Muneyuki, E. \& Yoshida, M. An alternative reaction pathway of $\mathrm{F}_{1}$-ATPase suggested by rotation without 80 degrees/40 degrees substeps of a sluggish mutant at low ATP. Biophys. J. 90, 1028-1032 (2006).

22. Watanabe, R. et al. Mechanical modulation of catalytic power on $F_{1}$-ATPase. Nat. Chem. Biol. 8, 86-92 (2012).

23. Nitta, R., Kikkawa, M., Okada, Y. \& Hirokawa, N. KIF1A alternately uses two loops to bind microtubules. Science 305, 678-683 (2004).

24. Yount, R. G., Lawson, D. \& Rayment, I. Is myosin a 'back door' enzyme? Biophys. J. 68, 44S-49S (1995).

25. Dittrich, M., Hayashi, S. \& Schulten, K. ATP hydrolysis in the $\beta_{\mathrm{TP}}$ and $\beta_{\mathrm{DP}}$ catalytic sites of $\mathrm{F}_{1}$-ATPase. Biophys. J. 87, 2954-2967 (2004).

26. Yoshida, M., Poser, J. W., Allison, W. S. \& Esch, F. S. Identification of an essential glutamic acid residue in the beta subunit of the adenosine triphosphatase from the thermophilic bacterium PS3. J. Biol. Chem. 256, 148-153 (1981).

27. Al-Shawi, M. K., Parsonage, D. \& Senior, A. E. Thermodynamic analyses of the catalytic pathway of $\mathrm{F}_{1}$-ATPase from Escherichia coli. Implications regarding the nature of energy coupling by $\mathrm{F}_{1}$-ATPases. J. Biol. Chem. 265, 4402-4410 (1990).

28. Amano, T., Tozawa, K., Yoshida, M. \& Murakami, H. Spatial precision of a catalytic carboxylate of $\mathrm{F}_{1}$-ATPase beta subunit probed by introducing different carboxylate-containing side chains. FEBS Lett. 348, 93-98 (1994).

29. Enoki, S., Watanabe, R., Iino, R. \& Noji, H. Single-molecule study on the temperature-sensitive reaction of $\mathrm{F}_{1}$-ATPase with a hybrid $\mathrm{F}_{1}$ carrying a single B(E190D). J. Biol. Chem. 284, 23169-23176 (2009).

30. Watanabe, R., Hayashi, K., Ueno, H. \& Noji, H. Catalysis-enhancement via rotary fluctuation of $\mathrm{F}_{1}$-ATPase. Biophys. J. 105, 2385-2391 (2013).

31. Spetzler, D. et al. Single molecule measurements of $F_{1}$-ATPase reveal an interdependence between the power stroke and the dwell duration. Biochemistry 48, 7979-7985 (2009).

32. Hirono-Hara, Y. et al. Pause and rotation of $\mathrm{F}_{1}$-ATPase during catalysis. Proc. Natl Acad. Sci. USA 98, 13649-13654 (2001).

33. Adachi, K., Oiwa, K., Yoshida, M., Nishizaka, T. \& Kinosita, K. Controlled rotation of the $\mathrm{F}_{1}$-ATPase reveals differential and continuous binding changes for ATP synthesis. Nat. Commun. 3, 1022 (2012).

34. Mitome, N. et al. The presence of phosphate at a catalytic site suppresses the formation of the MgADP-inhibited form of $\mathrm{F}_{1}$-ATPase. Eur. J. Biochem. 269, 53-60 (2002)

35. Milgrom, Y. M. \& Boyer, P. D. The ADP that binds tightly to nucleotidedepleted mitochondrial $\mathrm{F}_{1}$-ATPase and inhibits catalysis is bound at a catalytic site. Biochim. Biophys. Acta 1020, 43-48 (1990). 
36. Hyndman, D. J., Milgrom, Y. M., Bramhall, E. A. \& Cross, R. L. Nucleotide-binding sites on Escherichia coli $F_{1}$-ATPase. Specificity of noncatalytic sites and inhibition at catalytic sites by MgADP. J. Biol. Chem. 269, 28871-28877 (1994).

37. Hirono-Hara, Y., Ishizuka, K., Kinosita, Jr. K., Yoshida, M. \& Noji, H. Activation of pausing $\mathrm{F}_{1}$ motor by external force. Proc. Natl Acad. Sci. USA 102, 4288-4293 (2005).

38. Bowler, M. W., Montgomery, M. G., Leslie, A. G. \& Walker, J. E. How azide inhibits ATP hydrolysis by the F-ATPases. Proc. Natl Acad. Sci. USA 103, 8646-8649 (2006).

39. Rees, D. M., Montgomery, M. G., Leslie, A. G. \& Walker, J. E. Structural evidence of a new catalytic intermediate in the pathway of ATP hydrolysis by $\mathrm{F}_{1}$-ATPase from bovine heart mitochondria. Proc. Natl Acad. Sci. USA 109, 11139-11143 (2012).

40. Menz, R. I., Walker, J. E. \& Leslie, A. G. Structure of bovine mitochondrial $F_{1}$-ATPase with nucleotide bound to all three catalytic sites: implications for the mechanism of rotary catalysis. Cell 106, 331-341 (2001).

41. Bald, D. et al. The noncatalytic site-deficient $\alpha_{3} \beta_{3} \gamma$ subcomplex and $\mathrm{F}_{\mathrm{o}} \mathrm{F}_{1}$-ATP synthase can continuously catalyse ATP hydrolysis when $\mathrm{P}_{\mathrm{i}}$ is present. Eur. J. Biochem. 262, 563-568 (1999).

42. Watanabe, R. et al. Biased Brownian stepping rotation of $\mathrm{F}_{\mathrm{o}} \mathrm{F}_{1}$-ATP synthase driven by proton motive force. Nat. Commun. 4, 1631 (2013).

43. Diez, M. et al. Proton-powered subunit rotation in single membrane-bound $\mathrm{F}_{\mathrm{o}} \mathrm{F}_{1}$-ATP synthase. Nat. Struct. Mol. Biol. 11, 135-141 (2004).

44. Duser, M. G. et al. 36 degrees step size of proton-driven c-ring rotation in $\mathrm{F}_{\mathrm{o}} \mathrm{F}_{1}$-ATP synthase. EMBO J. 28, 2689-2696 (2009).

\section{Acknowledgements}

We thank all members of Noji Laboratory for technical support. This work was supported by Grants-in-Aid for Scientific Research (No. 18074005) to H.N. and (No. 30540108) to R.W. from the Ministry of Education, Culture, Sports, Science, and Technology, Japan.

\section{Author contributions}

R.W. designed and performed experiments and analysed data; H.N. designed experiments, built whole story and wrote papers with R.W.

\section{Additional information}

Competing financial interests: The authors declare no competing financial interests.

Reprints and permission information is available online at http://npg.nature.com/ reprintsandpermissions/

How to cite this article: Watanabe, R. et al. Timing of inorganic phosphate release modulates the catalytic activity of ATP-driven rotary motor protein. Nat. Commun. 5:3486 doi: 10.1038/ncomms4486 (2014).

(C) $\Theta$ This work is licensed under a Creative Commons AttributionNonCommercial-NoDerivs 3.0 Unported License. The images or other third party material in this article are included in the article's Creative Commons license, unless indicated otherwise in the credit line; if the material is not included under the Creative Commons license, users will need to obtain permission from the license holder to reproduce the material. To view a copy of this license, visit http://creativecommons.org/licenses/by-nc-nd/3.0/ 